\title{
High Electron Mobility InN
}

R.E. Jones, S.X. Li, E.E. Haller

Materials Sciences Division, Lawrence Berkeley National Laboratory and Dept. of Materials

Science and Engineering, University of California, Berkeley, CA 94720 USA

H.C.M. van Genuchten, K.M. Yu, J.W. Ager III, Z. Liliental-Weber, W. Walukiewicz

Materials Sciences Division, Lawrence Berkeley National Laboratory, Berkeley, CA 94720 USA

H. Lu and W.J. Schaff

Dept. of Electrical Engineering and Computer Science, Cornell University, Ithaca, NY 14853

USA

\begin{abstract}
Irradiation of $\mathrm{InN}$ films with $2 \mathrm{MeV} \mathrm{He}^{+}$ions followed by thermal annealing below $500^{\circ} \mathrm{C}$ creates films with high electron concentration and mobility, as well as strong photoluminescence. Calculations show that electron mobility in irradiated samples is limited by triply charged donor defects. Subsequent thermal annealing removes a fraction of the defects, decreasing the electron concentration. There is a large increase in electron mobility upon annealing; the mobilities approach those of the as-grown films, which have 10 to 100 times smaller electron concentrations. Spatial ordering of the triply charged defects is suggested to cause the unusual increase in electron mobility.
\end{abstract}


Significant progress has been made recently in understanding the electronic properties of InN [1]. InN has the highest electron affinity of any known semiconductor, and the tendency for n-type doping in the material has been explained by this extremely low position of the conduction band edge with respect to the Fermi level stabilization energy $\left(E_{\mathrm{FS}}\right)$, along with a large concentration of point defects [2]. Still, electron transport in crystalline InN films is not fully understood. In this letter, we study the effect of $2 \mathrm{MeV} \mathrm{He}^{+}$irradiation followed by thermal annealing on the electronic properties of InN. We show that this process creates films with high electron concentrations and high electron mobilities, suggesting it as an effective technique for n-type doping.

According to the amphoteric defect model, the type (donor or acceptor) of the dominant native defects in a material depends on the location of the Fermi energy $\left(E_{\mathrm{F}}\right)$ relative to the common energy reference, $E_{\mathrm{FS}}$, which is located $4.9 \mathrm{eV}$ below the vacuum level [3]. When $E_{\mathrm{F}}<$ $E_{\mathrm{FS}}\left(E_{\mathrm{F}}>E_{\mathrm{FS}}\right)$, the formation energy of charged donor (acceptor) defects is reduced, and therefore native defects in a material tend to be donors (acceptors). $E_{\mathrm{F}}$ is stabilized when $E_{\mathrm{F}}=$ $E_{\mathrm{FS}}$, i.e., the defect creation rates are the same for donors and acceptors. In the unique case of InN with $E_{\mathrm{FS}}$ lying high in the conduction band, native donor defects are dominant, except in the cases of extremely high extrinsic doping (not considered here). As a consequence, in InN, the free electron concentration increases with increasing irradiation fluence until it saturates when $E_{\mathrm{F}}$ $=E_{\mathrm{FS}}[2]$.

The InN films $(1.8 \mu \mathrm{m})$ used in this study were grown by molecular beam epitaxy on csapphire substrates with an AIN and/or GaN buffer layer, and were nominally undoped [4]. Electron concentrations in the as-grown films ranged from $6 \times 10^{17}$ to $1 \times 10^{18} \mathrm{~cm}^{-3}$, with 
corresponding electron mobilities from 1100 to $500 \mathrm{~cm}^{2} / \mathrm{Vs}$. In order to predictably generate native point defects, the films were irradiated with $2 \mathrm{MeV} \mathrm{He}^{+}$ions. The irradiation fluences ranged from $1.1 \times 10^{14}$ to $8.9 \times 10^{15} \mathrm{~cm}^{-2}$. The irradiation produced nearly uniform damage across the film thickness and the end of range for the $2 \mathrm{MeV} \mathrm{He}^{+}$ions was in the substrate.

Rapid thermal annealing was performed sequentially on each sample to eliminate effects of inhomogeneity between samples. A Heatpulse 10T-02 Rapid Thermal Pulsing System with flowing $\mathrm{N}_{2}$ gas was used. The films were annealed first at $375^{\circ} \mathrm{C}$ and $/$ or $425^{\circ} \mathrm{C}$ for increasing time intervals from 10 to 300 seconds. Subsequent annealing was performed for the same increasing time intervals at $475^{\circ} \mathrm{C}$. Annealing temperatures of $500^{\circ} \mathrm{C}$ and higher caused film delamination, as did repeated annealing for times longer than one hour at $475^{\circ} \mathrm{C}$. The onset of delamination determined the endpoint of the annealing treatments. No data was used from films once delamination began to occur.

The electrical properties of the films were measured at room temperature by the Hall effect with a 3000 Gauss magnet, using indium contacts in the van der Pauw configuration. The contacts were removed prior to annealing by etching in $\mathrm{HCl}$. Photoluminescence (PL) signals were generated in the backscattering geometry with excitation by the $515 \mathrm{~nm}$ line of an argon laser. The signals were dispersed by a one meter double-grating monochromator and detected by a liquid-nitrogen-cooled germanium photodiode.

High-energy particle irradiation has been shown to be an effective method for controlling the electronic properties in $\mathrm{InN}$ films $[2,5,6]$. There is a linear relationship between electron concentration $(n)$ and $2 \mathrm{MeV} \mathrm{He}^{+}$ion fluence, with an electron production rate of about $4 \times 10^{4}$ electrons (ion-cm) ${ }^{-1}$ up to a saturation concentration of $\sim 4 \times 10^{20} \mathrm{~cm}^{-3}$. Further, modeling of electron mobility $(\mu)$ vs. $n$ data has shown that $\mu$ in irradiated films is controlled by scattering by 
triply charged donor defects produced by the irradiation [5]. The energy-dependent $\mu$ limited by ionized defect scattering in a nonparabolic band can be expressed as $[5,8]$ :

$$
\mu_{i}(k)=\frac{\varepsilon_{0}^{2}}{2 \pi e^{3} \hbar Z^{2} N_{i} F_{i}}\left(\frac{d E}{d k}\right)^{2} k,
$$

where $\varepsilon_{0}$ is the static dielectric constant (a value of 9.3 was used [7]), $Z$ is the charge of the ionized defects, and $F_{i}$ is a $k$-dependent function that incorporates free electron screening effects and the reduction of the scattering rates due to the mixed nature of the conduction band wavefunctions $[5,8]$. In uncompensated material, as was assumed here, the concentration of ionized defects $\left(N_{i}\right)$ is given by $n / Z$.

In analogy with the GaAs case [3], we suggest that the triply charged donor defects produced by irradiation are nitrogen vacancies $\left(V_{\mathrm{N}}\right)$ and relaxed indium vacancies $\left(V_{\text {In }}\right)$. In GaAs, when $E_{\mathrm{F}}$ lies below $E_{\mathrm{FS}}$, both vacancy sites act as donors. The Ga vacancy relaxes into an As antisite and an As vacancy, which form a triply charged donor complex. While $E_{\mathrm{F}}$ lies below $E_{\mathrm{FS}}$ only in p-type GaAs, it is below $E_{\mathrm{FS}}$ in all of the InN films considered here. Thus, both the $V_{\text {In }}$ and the $V_{\mathrm{N}}$ can be triply-charged donors, with the $V_{\text {In }}$ relaxing via the reaction: $V_{\text {In }} \rightarrow\left(\mathrm{N}_{\text {In }}+\right.$ $\left.V_{\mathrm{N}}\right)^{3+}$.

Figure 1 shows $\mu$ in the InN films as-grown, after irradiation, and after the subsequent annealing, as a function of $n$. The values calculated using Eq. 1 for $\mu$ limited by triply or singly charged donor defect scattering are also shown. It can be seen that $\mu$ after irradiation can be well explained by scattering from triply charged donors. Rapid thermal annealing of the irradiated films causes a decrease in $n$ and an increase in $\mu ; \mu$ increases up to a factor of four and $n$ is roughly halved from the values after irradiation. This increase in $\mu$ is especially striking because the values of the irradiated and annealed films are close to those of the as-grown films that have 
more than an order of magnitude lower $n$. It should be noted that, as shown in Fig. 1, this behavior is not seen in films that were annealed without first being subjected to irradiation.

Thermal annealing of the irradiated InN films also causes a recovery of the PL. As shown in the inset of Fig. 2, at the lowest fluences used in this work, $2 \mathrm{MeV} \mathrm{He}^{+}$irradiation causes a slight increase in PL intensity, due to the increased $n$. At higher fluences, the PL is gradually quenched as $E_{\mathrm{F}}$ approaches the donor defect levels located near $E_{\mathrm{FS}}$, which become efficient electron traps [6]. Upon thermal annealing after irradiation, the PL recovers (Fig. 2), and the recovery is consistent with the change in electrical properties discussed above.

Surprisingly, the increase in $\mu$ and decrease in $n$ cannot be fully explained by the removal of the defects created by irradiation. The simple removal of defects would be expected to create films with properties similar to those irradiated with lower fluences. However, as seen in Fig. 3, $\mu$ after annealing is not described by the theoretical $\mu$ limit for triply charged defect scattering, which well describes the irradiated films. After the initial annealing treatments, $\mu$ increases above this theoretical line. Further increases in $\mu$ are observed after longer annealing treatments and treatments at higher temperatures. In fact, the values of $\mu$ approach the theoretical limit for singly charged defect scattering, suggesting that two types of defects may be created by the irradiation: triply charged defects that are removed by annealing, and singly charged defects that are stable upon annealing and that therefore limit $\mu$ in annealed films.

We modeled the change in $\mu$ with annealing assuming that the irradiation created singly and triply charged defects, but that annealing removed only triply charged defects, in order to realize the largest increase in $\mu$. The rate of defect removal was related to the decrease in $n$; one triply charged defect was removed for every decrease of three electrons. The starting ratio of triply to singly charged defects was varied widely to find the best fit to the experimental results. 
However, for no ratio was there a reasonable fit between the experiment and model. Figure 3 shows the modeled electrical properties for two samples assuming that irradiation creates defects in the ratio of three singly to two triply charged defects, and that annealing removes the triply charged defects. It can be seen that both the absolute values as well as the slope of the calculated $\mu$ are a poor fit to the experimental data. The experimental $\mu$ increase much faster than what would be predicted due to the removal of triply charged defects. This is especially evident in the sample with lower $n$; for a range of annealing steps, a considerable increase in $\mu$ is observed with an almost constant $n$ of about $3 \times 10^{19} \mathrm{~cm}^{-3}$. Similar behavior was seen in all samples in which $n$ fell below $5 \times 10^{19} \mathrm{~cm}^{-3}$.

It also can be shown that this unusual increase in $\mu$ cannot be accounted for by any compensation scheme. As $n$ decreases, the compensation would necessarily increase; therefore $\mu$, which is limited by ionized defect scattering, could not have the observed increase. Instead, we suggest that the change in electrical properties results from a combination of annealinginduced defect removal and spatial ordering of the remaining triply charged defects due to the repulsive Coulomb interaction between them. It has been well recognized that ordering of dopants could offer a unique opportunity to reduce ionized impurity scattering, improving charge carrier mobilities and the performance of electronic devices [9]. However, previous attempts to order B acceptors in Si were not fully successful [10], and no improvement in the carrier mobilities in ordered impurity systems was ever observed. The reason for the difficulties in ordering of intentionally introduced impurities is that there is only a weak Coulomb repulsion between singly charged centers. In contrast, the Coulomb repulsion energy is nine times larger for triply charged centers, as exist in our system. This is expected to greatly increase the transition temperature from the ordered to disordered state. 
In order to evaluate the range of the critical temperatures for the defect ordering to occur, we extended the theory developed for ordering of electrons on defect sites [11]. We calculated the critical temperature below which a lattice of ordered triply charged defects is lower in energy than a random distribution. For the range of defect concentrations in our study, the critical temperature ranges between $100-400 \mathrm{~K}$, suggesting that a correlation of defect positions may be energetically favorable at room temperature. As a comparison, the temperature range would be $25-100 \mathrm{~K}$ for a system of singly charged defects corresponding to the same $n$. After irradiation, the defects are randomly distributed and they do not have sufficient energy to move into equilibrium positions. Thermal annealing in the range of $375-475^{\circ} \mathrm{C}$ provides sufficient energy for defect diffusion, and upon cooling, the defects form a correlated equilibrium state due to the Coulomb repulsion between them.

Evidence that defect diffusion occurs during thermal annealing is shown in Figure 4. Transmission electron micrographs of the films after irradiation and annealing show that $9 \times 10^{10}$ $3 \times 10^{11} \mathrm{~cm}^{-2}$ dislocation loops with diameters of 5-20 nm exist in the areas free of threading dislocations, in addition to small voids observed along the threading defects. The loops appear to be formed by the agglomeration of individual vacancies that were created by the irradiation. In samples that were annealed but not first irradiated, there are larger voids along the threading defects, but no dislocation loops in defect free areas. The samples which were only irradiated do not show voids along dislocation lines or a comparable number of dislocation loops.

The equilibrium state arrived at after annealing cannot be a perfect, long range superlattice of defects, due to the presence of dislocations and other extended defects that introduce random potentials. However it has been shown that even partial ordering will significantly reduce the effectiveness of the scattering and lead to an enhancement in $\mu$ [11]. It is 
important to note that the ordering does not affect the number of donor defects and thus $n$ is unchanged. Unlike any compensation or selective defect annealing scheme, the ordering well accounts for the experimentally observed increase in $\mu$ at almost constant $n$ seen in samples with lower $n$.

In summary, irradiation and thermal annealing of $\mathrm{InN}$ films has been shown to produce material with both high electron concentration and high electron mobility, and thus may be considered to be a method for controlled n-type doping of InN. The irradiation creates triply charged donor defects, some of which are removed by annealing. The experimental mobilities may be further explained by a partial ordering of the remaining defects, which reduces their electron scattering efficiency. Recovery of the photoluminescence upon annealing also indicates that the films have improved transport properties. This may be the first evidence in a semiconductor for a spatial correlation of ionized defects themselves, rather than of electrons on a defect lattice.

\section{ACKNOWLEDGMENTS}

This work is supported by the Director, Office of Science, Office of Basic Energy Sciences, Division of Materials Sciences and Engineering, of the U.S. Department of Energy under Contract No. DE-AC02-05CH11231. The work at Cornell University is supported by ONR under Contract No. N000149910936. One of the authors (REJ) thanks the U.S. Department of Defense for support in the form of a NDSEG fellowship.

\section{REFERENCES}

[1] W. Walukiewicz, J. W. Ager III, K. M. Yu, Z. Liliental-Weber, J. Wu, S. X. Li, R. E. Jones, and J. D. Denlinger, J. Phys. D: Appl. Phys. 39 R83 (2006). 
[2] S.X. Li, K.M. Yu, J. Wu, R.E. Jones, W. Walukiewicz, J.W. Ager III, W. Shan, E.E. Haller, H. Lu, and W. J. Schaff, Phys. Rev. B 71 (16), 161201 (2005).

[3] W. Walukiewicz, Physica B, 302, 123 (2001).

[4] H. Lu, W. J. Schaff, L. F. Eastman, and C. Wood, Mat. Res. Soc. Symp. Proc. 693, I1.5 (2002).

[5] R.E. Jones, S.X. Li, L. Hsu, K.M. Yu, W. Walukiewicz, Z. Liliental-Weber, J.W. Ager III, E.E. Haller, H. Lu and W.J. Schaff, Physica B 376-377, 436 (2006).

[6] S.X. Li, R. E. Jones, E. E. Haller, K. M. Yu, W. Walukiewicz, J. W. Ager III, Z. LilientalWeber, H. Lu, and W. J. Schaff, Appl. Phys. Lett. 88, 151101 (2006).

[7] V.Y. Davydov and A. A. Klochikhin, Semiconductors 38, 897 (2004).

[8] W. Zawadzki and W. Szymanska, Phys. Stat. Sol. (b) 45, 415 (1971).

[9] A.F.J. Levi, S.L. McCall and P.M. Platzman, Appl. Phys. Lett. 54, 940 (1989).

[10] B.E. Weir, R.L. Headrick, Q. Shen, L.C. Feldman, M.S. Hybertsen, M. Needels, M.

Schluter, and T.R. Hart, Phys. Rev. B 46, 12861 (1992).

[11] J. Mycielski, Sol. St. Comm. 60, 165 (1986). 
Figure Captions

Figure 1: Electrical properties of the films used in this study, as-grown and after irradiation and/or thermal annealing treatments. Theoretical $\mu$ limited by singly and triply charged donor defect scattering is also shown. In the irradiated films, the higher $n$ correspond to higher $2 \mathrm{MeV}$ $\mathrm{He}^{+}$fluences.

Figure 2: PL signal of one InN film after irradiation with $1.1 \times 10^{15} \mathrm{He}^{+} \mathrm{cm}^{-2}$ and then successive thermal annealing treatments. The inset shows the PL of an InN film after increasing irradiation fluences; the signal is modulated by Fabry-Perot oscillations. The arrow relates the two films with the same irradiation fluences.

Figure 3: $\mu$ as a function of $n$ in two InN films after each successive thermal annealing treatment. The lines were calculated by assuming that irradiation produces a 3:2 ratio of singly to triply charged defects, and that the only effect of thermal annealing is to remove triply charged donor defects. The poor correspondence between the lines and the experimental data is evidence for spatial ordering of the remaining defects.

Figure 4: Cross-section micrograph of one InN film after irradiation with $8.9 \times 10^{15} \mathrm{He}^{+} \mathrm{cm}^{-2}$ and then thermal annealing. The concentration of dislocation loops with size between 5-20 nm is $9 \times 10^{10} \mathrm{~cm}^{-2}$; as an example, four of the loops are marked by arrows. The AlN/GaN buffer layer and sapphire substrate are also shown. 


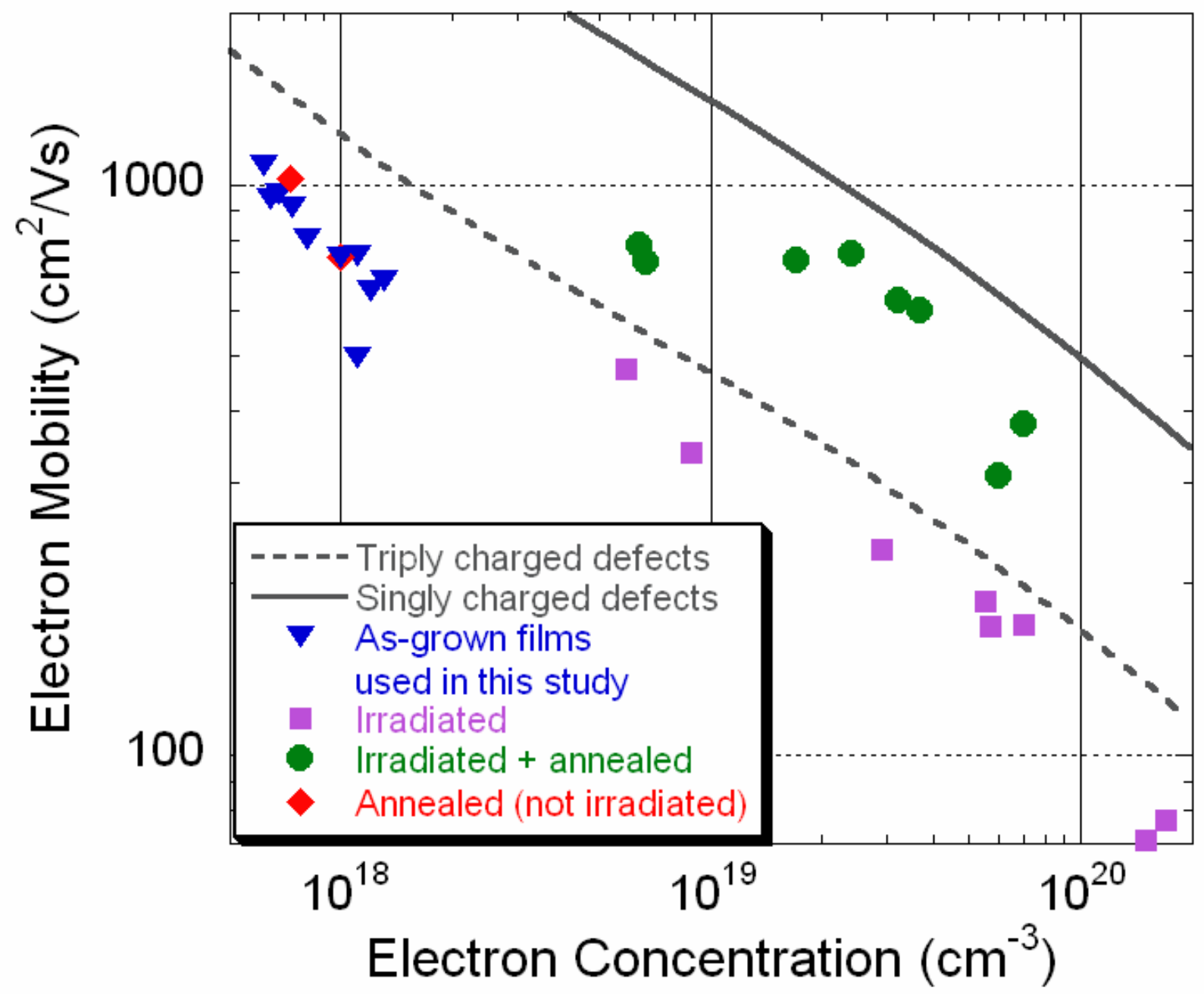

Figure 1 


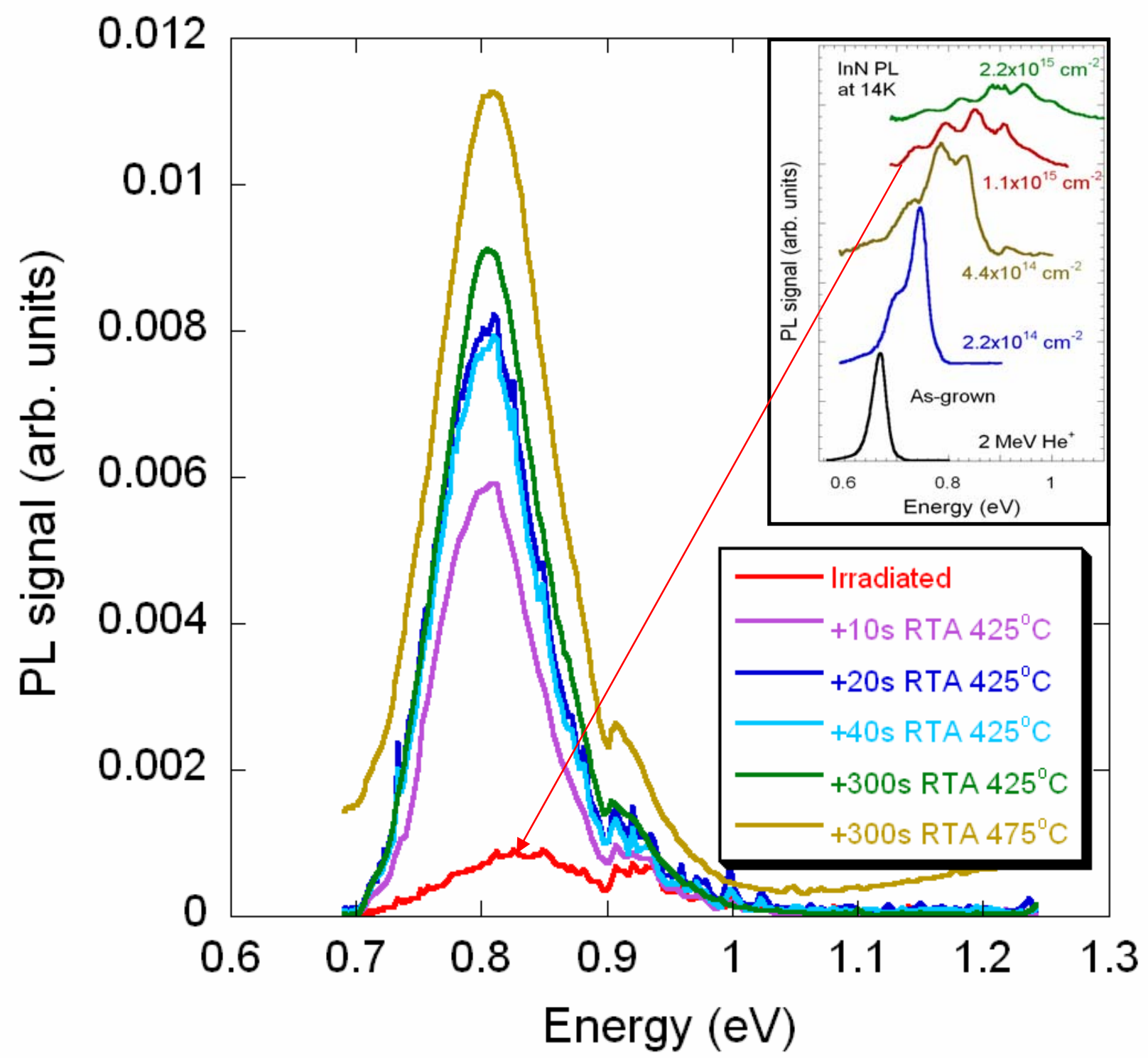

Figure 2 

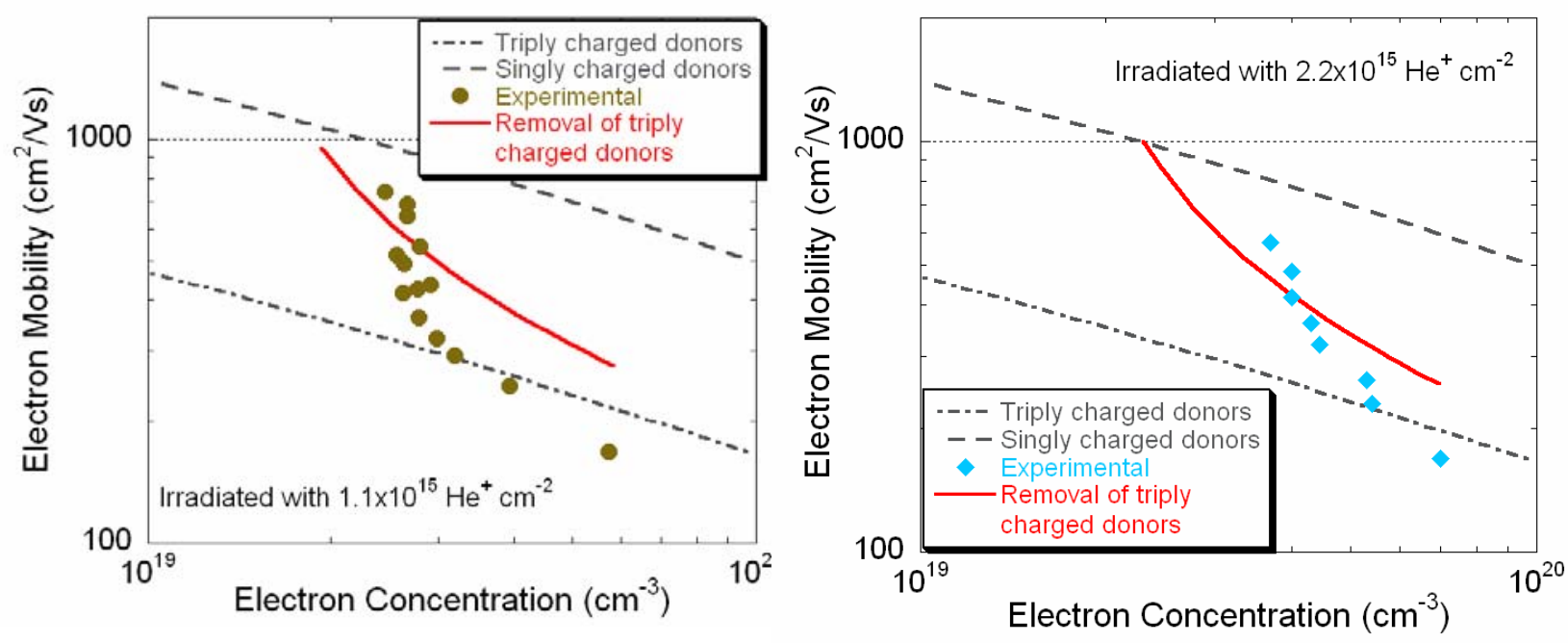

Figure 3 


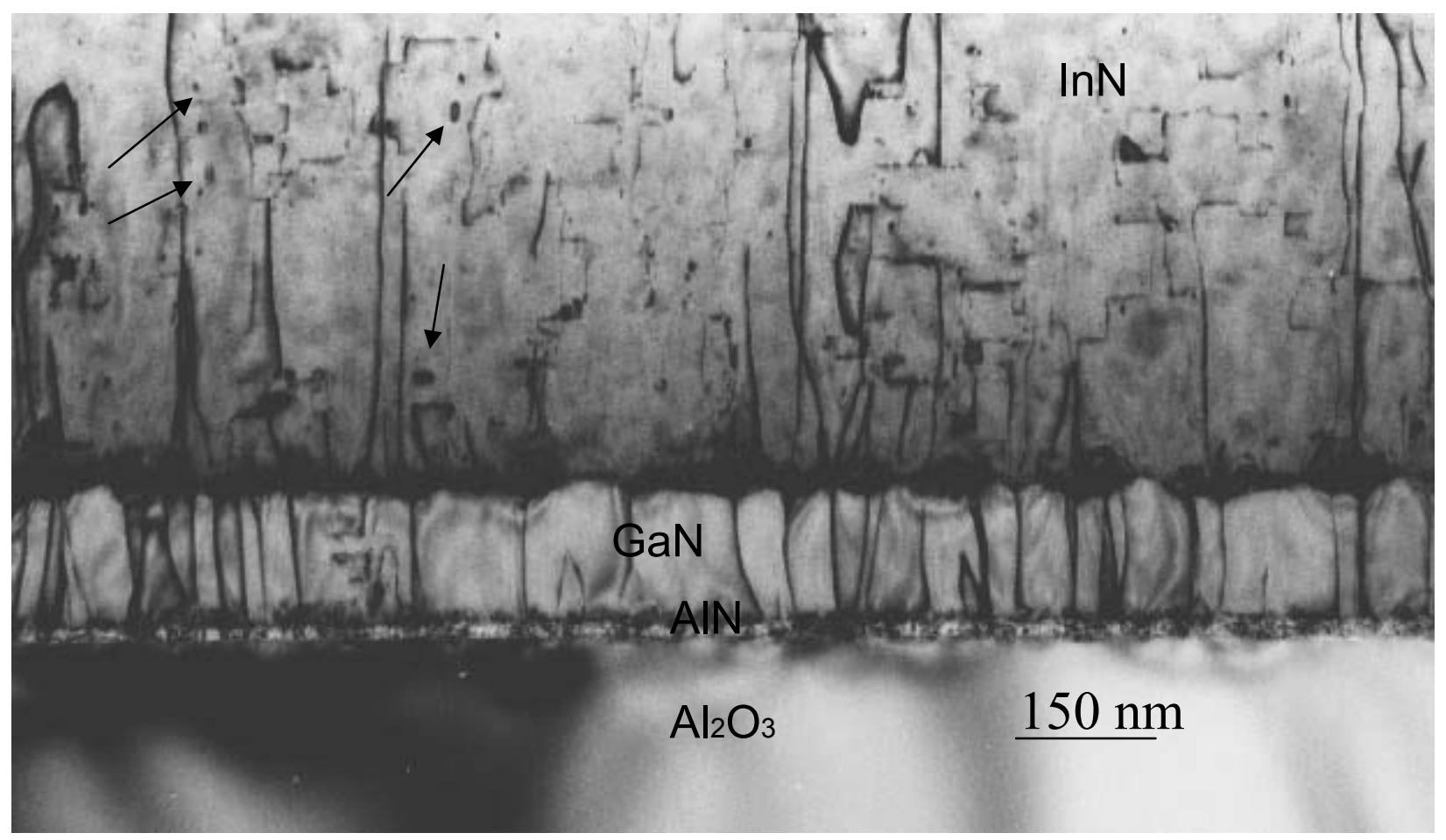

Figure 4 